\title{
Surgical and Functional Outcomes on Omitting Cortical Renorrhaphy During Robot-Assisted Versus Laparoscopic Partial Nephrectomy: A Match-Paired Analysis
}

Masashi Kubota ( $\nabla$ maskubo@kuhp.kyoto-u.ac.jp )

Kobe City Medical Center General Hospital

Toshinari Yamasaki

Kobe City Medical Center General Hospital

Shiori Murata

Kobe City Medical Center General Hospital

Yohei Abe

Kobe City Medical Center General Hospital

Yoichiro Tohi

Kobe City Medical Center General Hospital

\section{Yuta Mine}

Kobe City Medical Center General Hospital

Hiroki Hagimoto

Kobe City Medical Center General Hospital

Hidetoshi Kokubun

Kobe City Medical Center General Hospital

Issei Suzuki

Kobe City Medical Center General Hospital

Naofumi Tsutsumi

Kobe City Medical Center General Hospital

Koji Inoue

Kobe City Medical Center General Hospital

Mutsushi Kawakita

Kobe City Medical Center General Hospital

\section{Research Article}

Keywords: Nephron-sparing surgery, Partial nephrectomy, Suture, Renorrhaphy, Robot-assisted

Posted Date: January 3rd, 2022 
DOI: https://doi.org/10.21203/rs.3.rs-1191585/v1

License: (c) (1) This work is licensed under a Creative Commons Attribution 4.0 International License. Read Full License 


\section{Abstract \\ Objectives}

To assess surgical and functional outcomes in comparison of cortical renorrhaphy omitting, robotassisted partial nephrectomy (CRO-RAPN), and laparoscopic partial nephrectomy (CRO-LPN).

\section{Methods}

Between July 2012 and June 2020, patients with localized clinical T1-2 renal masses who underwent CRO-RAPN or CRO-LPN were reviewed. The outcomes of the CRO-RAPN and CRO-LPN groups were compared using propensity score matching. Trifecta was defined as negative surgical margin, less than 25 minutes of warm ischemic time, and no complications of Clavien-Dindo grade III or more until three months postoperatively. Preservation rate of the estimated glomerular filtration rate (eGFR) was evaluated at six months postoperatively.

\section{Results}

A total of 291 patients, including 210 patients who underwent CRO-RAPN and 81 patients who underwent CRO-LPN, were included, and matched pairs of 150 patients were analyzed. The CRO-RAPN group was associated with a significantly shorter warm ischemic time (13 $\mathrm{min}$ vs $20 \mathrm{~min}, P<0.001$ ), shorter total operation time (162 min vs $212 \mathrm{~min}, P<0.001)$, less estimated blood loss ( $40 \mathrm{~mL}$ vs $119 \mathrm{~mL}, P=0.002$ ), lower incidence of overall complications ( $3 \%$ vs $16 \%, P=0.001$ ), higher preservation rate of eGFR at six months postoperatively ( $93 \%$ vs $89 \%, P=0.003)$, and higher trifecta achievement rate $(84 \%$ vs $64 \%, P=$ 0.004) than the CRO-LPN group.

\section{Conclusions}

CRO-RAPN contributes to a shorter warm ischemic time, less blood loss, fewer complications, and preservation of renal function and makes it feasible to achieve a higher rate of trifecta compared to CROLPN.

\section{Introduction}

Partial nephrectomy (PN) is the gold standard for definitive therapy of T1 renal masses when surgery is indicated ${ }^{(1),(2)}$. Robot-assisted partial nephrectomy (RAPN) and laparoscopic partial nephrectomy (LPN) have progressed as minimally invasive PN and have expanded their indications to cases of complex and challenging renal tumors in recent years ${ }^{(3)},(4)$. In the early LPN series, suturing renorrhaphy of renal cortex layer was considered an indispensable procedure for ensuring hemostasis and closure of the 
urinary collecting system to avoid postoperative complications. In recent years, excessive renorrhaphy has been recognized as a concern since an injured vascularized parenchyma prevents preservation of postoperative renal function ${ }^{(5),(6)}$. Although a consensus regarding the optimal renorrhaphy technique for postoperative renal function preservation has not yet been established, the single-layer renorrhaphy technique has been considered to be favorable for postoperative renal function compared to the doublelayer renorrhaphy technique ${ }^{(7),(8)}$. Furthermore, omission of a cortical suture layer has recognized as an ideal approach to reduce the risk of complications and preserve healthy renal parenchyma (5), (9-12). However, the optimal platform to safely perform this challenging procedure has not been established. Previous studies have indicated that RAPN is more favorable than LPN in terms of renal functional preservation outcomes, shorter hospital stays, and shorter warm ischemia time ${ }^{(13)}$. These superiorities of RAPN compared with LPN are limited only among the conventional double-layer renorrhaphy technique. Therefore, this study aimed to investigate the impact of cortical renorrhaphy omitting-RAPN (CRO-RAPN) on surgical and functional outcomes compared to that of cortical renorrhaphy omitting-LPN (CRO-LPN).

\section{Methods}

\section{Study cohort and design}

This retrospective study included prospectively maintained data from an institutional database approved by our institutional review board. In this study, all patients registered in the database diagnosed with clinical T1-2 renal tumors who underwent PN between July 2012 and June 2020 were screened for possible retrospective analysis. The exclusion criteria of the study were patients who underwent open PN, solitary kidney, or recurrent and distant metastasis; showed bilateral tumor or multifocal tumor; and who received intraoperative cortical-layer renorrhaphy for any of the following reasons: (1) they decided to undergo cortical renorrhaphy preoperatively due to tumor complexity; or (2) they already registered in other clinical trials requiring intraoperative cortical-layer renorrhaphy. One group consisted of patients who underwent RAPN (CRO-RAPN group), and the other group underwent LPN (CRO-LPN group); the backgrounds and outcomes were compared between these two groups. The indications of RAPN or LPN were distinguished by the operation date: all patients who had received surgical procedures after April 2016 had been switched from LPN to RAPN since RAPN was approved by the Ministry of Health, Labour and Welfare and covered by the national health insurance.

To adjust for differences in baseline characteristics between patients who underwent RAPN and those who underwent LPN, propensity score matching was conducted. Propensity scores were calculated by logistic regression analysis with the dependent variable as the odds of undergoing LPN and the independent variables as age (years), sex (male vs female), body mass index $\left(\mathrm{kg} / \mathrm{m}^{2}\right)$, American Society of Anaesthesiologists physical status (ASA PS) (grades 1 to 2 vs 3 , and more), chronic kidney disease, Kidney Disease Improving Global Outcomes (KDIGO) classification ${ }^{(14)}$ (Grade 1 to 2 vs $3 a$ to $3 b$ vs 4 to 5), tumor side (right vs left), tumor size (mm), R.E.N.A.L. nephrometry score (4 to 6 vs 7 to 9 vs 10 to 12), and off-cramp procedure (on-cramp vs off-cramp). Patient characteristics of the RAPN and LPN groups 
were matched in a one-to-one fashion according to the propensity score. A caliper width of 0.2 , for the standard deviation, was applied.

\section{Surgical procedure}

Intraoperative procedures were standardized and were not modified for each group. Laparoscopic PN was performed using four or five ports and RAPN was performed using a four-arm da Vinci Si, $\mathrm{X}, \mathrm{Xi}^{\circledR}$-system (Intuitive Surgical, Sunnyvale, CA, USA) according to a previously reported procedure ${ }^{(12)}$. There were six ports, of which two were assistant surgeons. An additional port was required if liver elevation was necessary. No ureteral catheters were used for irrigation of the urinary tract. An intraoperative ultrasonography probe (L43K or L51K in RAPN, and Noblus in LPN; Hitachi, Tokyo, Japan) was used to identify the tumor location, size, depth, and flow. With ultrasound guidance, the resection margin was delineated. The renal artery was cramped using laparoscopic bulldog cramps. Tumor resection and hemostasis are shown in Figure 1. The renal capsule and cortical layer were cut with a margin of approximately 5-10 mm in LPN and 0-5 mm in RAPN, respectively. Upon reaching the layer of the parenchyma around the tumor, the tumor was bluntly dissected along with fiber lines of the renal parenchyma and resected with a thin margin to preserve the normal parenchyma as much as possible. During tumor resection, suction and soft coagulation (VIO 300D, ERBE Elektromedizin $\mathrm{GmbH}$ ) of a balltype electrode were performed by the assistant to control bleeding. The specimen was immediately placed in a Rusch Memobag (Teleflex, Morrisville, NC, USA). Single-layered inner running sutures ( $15 \mathrm{~cm}$ 3-0 V-Loc 180 V20; Covidien, New Haven, CT, USA) were performed only when entry into the collecting system or renal sinus was detected. For the inner sutures, the running needle was pierced at the inner edge of the renal parenchyma, and only minimal thin renal sinus tissue was sutured for closure. After inner suturing, the cramps (if used) were removed. After soft coagulation, absorbable hemostats (TachoSil; CSL Behring) were placed on the resection bed and manually pressed for a few minutes interposing the Rusch Memobag between TachoSil ${ }^{\circledR}$ and instruments. A single expert surgeon who had performed over 300 pure laparoscopic partial nephrectomies supervised a team of surgeons who were trained in the surgeries associated with this study.

\section{Data collection of clinical and surgical outcomes}

The extracted patient data from the prospective database included age, sex, body mass index, estimated glomerular filtration rate (eGFR), oncological background (clinical TNM stage, tumor side, tumor size, and R.E.N.A.L nephrometry score), perioperative information (ASA PS), total operation time, warm ischemic time, off-cramp technique, estimated blood loss, transfusions, surgeon, length of hospital stay, readmission, in-hospital complications, and Clavien-Dindo grade ${ }^{(15)}$. Regarding post-discharge complications, retrospective data were collected by reviewing the outpatient medical records.

The primary outcome was the achievement rate of trifecta, defined as a negative surgical margin, less than 25 minutes of warm ischemic time, and no complications of Clavien-Dindo grade III or more until three months postoperatively ${ }^{(16)}$. The secondary outcomes were as follows: total operation time, median 
warm ischemic time, intraoperative estimated blood loss, preservation rate of eGFR at six months postoperatively, stage upgrade of chronic kidney disease KDIGO grade, and the incidence of overall (Clavien-Dindo grade II or higher) complications at three months postoperatively. Bleeding-related complications included secondary procedures due to postoperative bleeding, transfusion, or intervention for postoperative hematoma infection.

\section{Statistical analysis}

A standard statistical software package (JMP®, ver. 13; SAS Institute, Chicago, IL) was used to conduct the statistical analyses. The Mann-Whitney $U$ and chi-square tests were used to determine the statistically significant between-group differences in the univariate analysis. Statistical significance was set at $P<$ 0.05 .

\section{Ethical approval}

All procedures performed in the present study involving human participants were in accordance with the ethical standards of the institutional research committee and with the 1964 Helsinki Declaration and its later amendments or comparable ethical standards. The study protocol was approved by Kobe City Medical Centre General Hospital institutional review board (IRB No. zn210405). The need for informed consent was waived by the institutional review committee due to the retrospective nature of the study.

\section{Results}

A flow diagram of the study is shown in Figure 2. Among the 336 consecutive patients screened, 40 patients were excluded based on the exclusion criteria. Among the 40 patients, twenty who underwent LPN and six who underwent RAPN in the initial phase decided to undergo cortical renorrhaphy preoperatively due to tumor complexity, and five participated in a trial that need cortical renorrhaphy in protocol. Five patients were lost to follow-up at six months postoperatively. Consequently, 291 patients, including 210 patients in the CRO-RAPN group and 81 patients in the CRO-LPN group, were finally included in this study and were followed up for at least six months postoperatively, and all were available for data collection with no missing data in terms of the required parameters.

As shown in Table 1, the patients in the CRO-RAPN group had significantly poorer eGFR values $\left(67 \mathrm{~mL} / \mathrm{min} / 1.73 \mathrm{~m}^{2}\right.$ vs $\left.73 \mathrm{~mL} / \mathrm{min} / 1.73 \mathrm{~m}^{2}, P=0.040\right)$ and higher R.E.N.A.L. nephrometry scores $(\geq$ $7: 56 \%$ vs $41 \%, P=0.047$ ) than those in the CRO-LPN group. The off-cramp procedure was indicated more for patients in the CRO-LPN group than in the RAPN group ( $6 \%$ vs $23 \%, P<0.001)$. However, there were no significant between-group differences in other baseline patient characteristics or surgical procedures. After matching analysis, 75 matched pairs were available for the final analysis. There were no significant differences in any of the baseline characteristics between the CRO-RAPN and CRO-LPN groups after pairmatched analysis. 
The perioperative outcomes before and after propensity matching between the CRO-RAPN and CRO-LPN groups are shown in Table 2. As the primary outcome, the comparison of trifecta achievement rate between the CRO-RAPN and CRO-LPN groups before and after propensity score matching was $79 \%$ vs $67 \%(P=0.027)$, and $84 \%$ vs $64 \%(P=0.004)$, respectively. Moreover, the median total operation time (162 min vs $212 \mathrm{~min}, P<0.001$ ), median warm ischemic time (13 min vs $20 \mathrm{~min}, P<0.001$ ), median estimated blood loss ( $40 \mathrm{~mL}$ vs $119 \mathrm{~mL}, P=0.002$ ), incidence of negative three-month overall complications ( $97 \%$ vs $84 \%, P=0.001$ ), median postoperative six-month decrease in eGFR $\left(5 \mathrm{~mL} / \mathrm{min} / 1.73 \mathrm{~m}^{2}\right.$ vs $\left.7 \mathrm{~mL} / \mathrm{min} / 1.73 \mathrm{~m}^{2}, P=0.002\right)$, achievement rate of at least $90 \%$ eGFR preservation ( $67 \%$ vs $48 \%, P=0.016)$, and the median six-month preservation rate of eGFR $(93 \%$ vs $89 \%$, $P=0.003)$ in the CRO-RAPN group were also significantly better than those in the CRO-LPN group after propensity score matching.

In the cohort before propensity score matching, two (1\%) patients underwent transcatheter arterial embolization due to postoperative bleeding in the CRO-RAPN group. Of these, one patient was admitted for postoperative urosepsis and a pseudoaneurysm was detected on the tumor bed on emergency computed tomography. Bleeding in the mesentery of the sigmoid colon was detected in the other patient due to intraoperative injury of the branch of the inferior mesenteric artery. However, one patient in the CRO-LPN group also needed transcatheter arterial embolization due to hemorrhagic shock from postoperative tumor bed bleeding. No other patient showed active bleeding or pseudoaneurysm on routine postoperative computed tomography. In the post-matching cohort, of the seven patients (9\%) who experienced bleeding-related complications in the CRO-LPN group, one (1\%) had postoperative bleeding, 3 (4\%) needed transfusion due to intraoperative bleeding, and $3(4 \%)$ required intravenous antibiotics due to infection of the perirenal hematoma. However, only one patient (1\%) needed intervention due to bleedingrelated complications in the CRO-RAPN group, and this incidence was significantly lower than that in the LPN group $(P=0.032)$.

\section{Discussion}

In this study, patients subjected to CRO-RAPN had a significantly shorter intraoperative warm ischemic time and lower incidence of complications than patients who underwent CRO-LPN. These results contributed to the improvement in trifecta achievement rates for CRO-RAPN. Moreover, higher preservation rate of postoperative renal function was showed in patients of CRO-RAPN compared with those of CRO-LPN. These facts were replicated in the cohort after propensity score matching, adjusting for patient background between the CRO-RAPN and CRO-LPN groups.

To our knowledge, this is the first study to evaluate the trifecta outcome of CRO-RAPN compared to those of CRO-LPN, and these were evaluated in one of the largest cohorts who underwent cortical renorrhaphy omitting-PN. Importantly, although prior studies have also shown that RAPN is superior to LPN in terms of estimated blood loss, warm ischemic time, and renal function preservation, at postoperative six months ${ }^{(13)}$, this study makes a significant contribution to the literature in that these results were replicated in a cohort limited to the omission of cortical renorrhaphy procedures. 
The robotic instruments improved the enhanced three-dimensional visualization of the operative field and increased degrees of freedom of powered wrists. This innovation makes it feasible to precisely close the resected renal sinus or urinary collecting system by inner suture that involves minimal renal parenchyma. Even in cases of small renal tumors that do not require an inner suture, bleeding control during resection with shorter warm ischemic times of robots may provide PN with accurate surgical margins and minimal volume loss of renal parenchyma. While it was also feasible with LPN, our results support that RAPN makes it easier to pursue to achieve such ideal PNs. Among the trifecta outcomes, the incidence of postoperative complications in the CRO-LPN group was significantly improved in the CRO-RAPN group. In particular, because the incidence of bleeding-related complications was superior in the CRO-RAPN group, the excellent controlled bleeding associated with the CRO-RAPN could ultimately have contributed to the improvement of the overall complication rate.

In this study, trifecta was utilized as the primary outcome for the comprehensive measurement of surgical quality and safety. Although several previous studies have propounded different definitions of trifecta of PN ${ }^{(16-19)}$, the definition that includes warm ischemic time as a trifecta factor was considered as appropriate for the evaluation of the challenging surgical technique of cortical renorrhaphy omitting-PN. Based on previous studies, the estimated goal for achievement rate of trifecta of RAPN is $68.0-82.6 \%$ (1619). Since the trifecta achievement rates of the RAPN and LPN groups after propensity score matching in this study were $84 \%$ and $64 \%$, respectively, our results show that it is possible to reach a sufficiently high achievement rate of trifecta with RAPN and LPN, even when omitting cortical renorrhaphy. Among previous studies, Hung et al. ${ }^{(19)}$ first proposed over $90 \%$ preservation of eGFR at six months postoperatively as an element of trifecta. However, associated with the trend of expanding the indications for PN to larger renal tumors, it is becoming increasingly difficult to achieve such a high eGFR preservation rate due to the large renal volume deficit. Indeed, the latest trifecta definition reported by Anceschi et al. ${ }^{(18)}$ set a goal of eGFR preservation of at least $70 \%$. Even in using the trifecta definition including $90 \%$ eGFR preservation cut-off established by Hung et al. a significant difference was also reproduced in the triad achievement rates between the RAPN and LPN groups after propensity score matching in this study ( $64 \%$ vs $43 \%, P=0.007$, respectively).

Our study has several limitations. First, this retrospective study involved a study population from a single institution that included a limited number of patients in the final analysis. Because we selected a composite outcome such as trifecta as the primary outcome of this study, we were unable to estimate which factors were directly involved in each outcome. In other words, it has not been clarified how decreased blood loss or shortened warm ischemic time directly contributes to the preservation of renal function. In addition, concerns about the learning curve could not be dispelled because indications of CRO-RAPN or CRO-LPN were divided by the date of institutional introduction of robotic systems in this study. A randomized prospective setting would be an efficacious design to resolve problems such as differences in surgical skills. Furthermore, data on the volume of the resected renal parenchyma could not be included in the analysis due to statistical complexity. The resection strategy was mainly used for resection in early LPN cases, while enucleoresection was mainly used in late RAPN cases (20), (21). The 
parameters of the surface-intermediate-base margin score ${ }^{(20),(21)}$ provide a value for the estimated volume loss of parenchyma and could be a solution to this problem in future studies. Despite several limitations, the present study showed that CRO-RAPN can be performed safely with a higher trifecta achievement rate compared to CRO-LPN. In omitting the cortical renorrhaphy procedure, RAPN makes it feasible to achieve shorter warm ischemic time, less blood loss, fewer complications, and decreased loss of renal function compared to LPN.

\section{Abbreviations}

ASA PS = American Society of Anesthesiologists physical status

eGFR= estimated glomerular filtration rate

LPN = laparoscopic partial nephrectomy

RAPN = robot-assisted radical partial nephrectomy

\section{Declarations}

\section{Acknowledgments}

The authors would like to acknowledge the assistance of the following people: Yoko Miyake, Ritsuki Yamaguchi, Noriyuki Makita, Ryosuke Suzuki, Yoshio Sugino, Kobe City Medical Centre General Hospital. Illustrations in Figure.1 were drawn by M.Kubota and Y.Miyake.

\section{Authour contributions}

Conception and design: M.K., T.Y. Acquisition of data: M.K., S.M., Y.A., Y.T., Y.M., H.H., H.K., I.S. Statistical analysis of data: M.K., T.Y., S.M., Y.A., Y.T. Drafting of the manuscript: M.K. Critical revision of the manuscript: T.Y., N.T., K.I., M.K. Supervision: M.K.

\section{Additional informaion}

All authors declare there are no competing interests associated with this study.

\section{References}

1. Ljungberg B, Albiges L, Abu-Ghanem Y, et al. European Association of Urology Guidelines on Renal Cell Carcinoma: The 2019 update. Eur Urol 2019; 75: 799-810. https://doi.org/10.1016/j.eururo.2019.02.011

2. Campbell S, Uzzo RG, Allaf ME, et al. Renal Mass and Localized Renal Cance: AUA guideline. J Urol 2017; 198: 520-9. https://doi.org/10.1016/j.juro.2017.04.100 
3. Buffi NM, Saita A, Lughezzani G, et al. Robot-assisted partial nephrectomy for complex (PADUA Score $\geq 10$ ) tumors: Techniques and results from a multicenter experience at four high-volume centers. Eur Urol 2020; 77: 95-100. https://doi.org/10.1016/j.eururo.2019.03.006

4. Verze $P$, Fedelini $P$, Chiancone $F$, et al. Perioperative and renal functional outcomes of laparoscopic partial nephrectomy (LPN) for renal tumours of high surgical complexity: A single-institute comparison between clampless and clamped procedures. World J Urol 2017; 35: 403-9. https://doi.org/10.1007/s00345-016-1882-7

5. Bahler CD, Sundaram CP. Effect of renal reconstruction on renal function after partial nephrectomy. J Endourol 2016; 30 Suppl 1: S37-S41. https://doi.org/10.1089/end.2016.0055

6. Porpiglia F, Bertolo R, Amparore D, Fiori C. Nephron-sparing suture of renal parenchyma after partial nephrectomy: Which technique to go for? Some Best Practices. Eur Urol Focus 2019; 5: 600-3. https://doi.org/10.1016/j.euf.2017.08.006

7. Bertolo R, Campi R, Klatte T, et al. Suture techniques during laparoscopic and robot-assisted partial nephrectomy: A systematic review and quantitative synthesis of peri-operative outcomes. BJU Int 2019; 123: 923-46. https://doi.org/10.1111/bju.14537

8. Bertolo R, Campi R, Mir MC, et al. Systematic review and pooled analysis of the impact of renorrhaphy techniques on renal functional outcome after partial nephrectomy. Eur Urol Oncol 2019; 2: 572-5. https://doi.org/10.1016/j.euo.2018.11.008

9. Bahler CD, Dube HT, Flynn KJ, et al. Feasibility of omitting cortical renorrhaphy during robot-assisted partial nephrectomy: A matched analysis. J Endourol 2015; 29: 548-55. https://doi.org/10.1089/end.2014.0763

10. Alrishan Alzouebi I, Williams A, Thiagarjan NR, Kumar M. Omitting cortical renorrhaphy in robotassisted partial nephrectomy: Is it safe? A single center large case series. J Endourol 2020; 34: 8406. https://doi.org/10.1089/end.2020.0121

11. Arora S, Bronkema C, Porter JR, et al. Omission of cortical renorrhaphy during robotic partial nephrectomy: A Vattikuti collective quality initiative database analysis. Urology 2020; 146: 125-32. https://doi.org/10.1016/j.urology.2020.09.003

12. Tohi $Y$, Murata S, Makita N, et al. Comparison of perioperative outcomes of robot-assisted partial nephrectomy without renorrhaphy: Comparative outcomes of cT1 a versus cT1b renal tumors. Int $\mathrm{J}$ Urol 2019; 26: 885-9. https://doi.org/10.1111/iju.14046

13. Choi JE, You JH, Kim DK, Rha KH, Lee SH. Comparison of perioperative outcomes between robotic and laparoscopic partial nephrectomy: A systematic review and meta-analysis. Eur Urol 2015; 67: 891-901. https://doi.org/10.1016/j.eururo.2014.12.028

14. Stevens PE, Levin A. Evaluation and management of chronic kidney disease: Synopsis of the kidney disease: Improving global outcomes 2012 clinical practice guideline. Ann Intern Med 2013; 158: 825-30. https://doi.org/10.7326/0003-4819-158-11-201306040-00007

15. Clavien PA, Barkun J, de Oliveira ML, et al. The Clavien-Dindo classification of surgical complications: Five-year experience. Ann Surg 2009; 250: 187-96. 
https://doi.org/10.1097/SLA.0b013e3181b13ca2

16. Khalifeh A, Autorino R, Hillyer SP, et al. Comparative outcomes and assessment of trifecta in 500 robotic and laparoscopic partial nephrectomy cases: A single surgeon experience. J Urol 2013; 189: $1236-42$.

17. Buffi N, Lista G, Larcher A, et al. Margin, ischemia, and complications (MIC) score in partial nephrectomy: A new system for evaluating achievement of optimal outcomes in nephron-sparing surgery. Eur Urol 2012; 62: 617-8. https://doi.org/10.1016/j.eururo.2012.06.001. https://doi.org/10.1016/j.juro.2012.10.021

18. Anceschi U, Ferriero MC, Tuderti G, et al. Head to head impact of Margin, Ischemia, Complications, Score Versus a novel Trifecta Score on oncologic and functional outcomes after robotic-assisted partial nephrectomy: Results of a multicenter series. Eur Urol Focus 2020; S2405-4569(20)30187-5. https://doi.org/10.1016/j.euf.2020.06.021

19. Hung AJ, Cai J, Simmons MN, Gill IS. "Trifecta" in partial nephrectomy. J Urol 2013; 189: 36-42. https://doi.org/10.1016/j.juro.2012.09.042

20. Minervini A, Carini M, Uzzo RG, Campi R, Smaldone MC, Kutikov A. Standardized reporting of resection technique during nephron-sparing surgery: The surface-intermediate-base margin score. Eur Urol 2014; 66: 803-5. https://doi.org/10.1016/j.eururo.2014.06.002

21. Minervini A, Campi R, Lane BR, et al. Impact of resection technique on perioperative outcomes and surgical margins after partial nephrectomy for localized renal masses: A prospective multicenter study. J Urol 2020; 203: 496-504. https://doi.org/10.1097/JU.0000000000000591

\section{Tables}


Table 1 Patient characteristics before and after propensity matching compared between the CRORAPN group and CRO-LPN group.

\section{Before propensity score matching}

Parameters

\section{Number of patients}

Median age, years $\left(\mathrm{IQR}^{+}\right)$

Male sex, $\mathrm{n}(\%)$

Median $\mathrm{BMI}^{\ddagger}, \mathrm{kg} / \mathrm{m}^{2}(\mathrm{IQR})$

ASA PS§ 3, or more, n (\%) 25 (12)

eGFR', $\mathrm{mL} / \mathrm{min} / 1.73 \mathrm{~m}^{2}$

$(\mathrm{IQR})$

KDIGO CKD ${ }^{\dagger+}$ grade, $\mathrm{n}(\%)$

Grade 1, or 2 (60

$\mathrm{mL} / \mathrm{min} / 1.73 \mathrm{~m}^{2}$, or more)

Grade 3a, or 3b (30-59

$\mathrm{mL} / \mathrm{min} / 1.73 \mathrm{~m}^{2}$ )

Grade 4, or 5 (29

$\mathrm{mL} / \mathrm{min} / 1.73 \mathrm{~m}^{2}$, or less)

Right side tumor, $\mathrm{n}(\%)$

Tumor size, mm (IQR)
97 (46)

$30(22-39)$

CRO- CRO-

$\mathrm{RAPN}^{\ddagger \ddagger}$ group LPN ${ }^{\S}$ group

210

$67(57-75) \quad 68(54-76)$

$67(55-77)$

$143(68)$

$58(28)$

$9(4)$

$2(2)$

$41(51)$

0.29

$29(23-37) \quad 0.84$
After propensity score matching

CRO- CRO-

RAPN LPN

group group

value

$75 \quad 75$

$67(57-\quad 68(52-\quad 0.77$

74) 75)

$49(65) \quad 46(61) \quad 0.37$

$\begin{array}{lll}23.6 & 24.3 & 0.39\end{array}$

(20.5- (22.0-

26.2) 26.0)

$11(15) \quad 7(9) \quad 0.23$

$68(59-\quad 73(56-\quad 0.31$

81) 85) 


\begin{tabular}{|c|c|c|c|c|c|c|}
\hline 10-12 (high) & $26(13)$ & $4(5)$ & & $2(3)$ & $4(5)$ & \\
\hline $\begin{array}{l}\text { Off-cramp procedure, } n \\
(\%)\end{array}$ & $13(6)$ & $19(23)$ & $<0.001$ & $12(16)$ & $13(17)$ & 0.50 \\
\hline
\end{tabular}

${ }^{\dagger}$ interquartile range; ${ }^{\ddagger}$ body mass index; $§$ American Society of Anesthesiologists physical status classification; "estimated glomerular filtration rate; ${ }^{\dagger+}$ chronic kidney disease; ${ }^{\ddagger \ddagger}$ cortical renorrhaphy omitting- robot-assisted partial nephrectomy; $\S^{\S}$ cortical renorrhaphy omitting- laparoscopic partial nephrectomy. 
Table 2 Surgical outcomes and complications before and after propensity matching compared between the CRO-RAPN group and CRO-LPN group.

Before propensity score matching

Parameters

$\begin{array}{lll}\text { CRO- } & \text { CRO- } & \text { p- } \\ \text { RAPN` group } & \text { LPN` group } & \text { value }\end{array}$

81

$169(144-$

196)

$16(11-23)$

Median war
min (IQR)

Median blood loss, $\mathrm{mL}(\mathrm{IQR})$

$40(15-90)$

119

$212(183-<0.001$
$238)$

$\min \left(\mathrm{IQR}^{\dagger}\right)$

210

$2(1)$

$134(64)$

$111(53)$

$3(4)$

$20(16-28)$

$<0.001$

(10-

$<0.001$

40

90)

$0.014 \quad 1(1)$

$3(4)$

0.31

Underwent inner suture, $\mathrm{n}(\%)$

Urinary tract entry, $\mathrm{n}(\%)$

7 (3)

$5(4-6)$

7 (3)

Overall (Clavien-Dindo grade

$\geq \mathrm{II})$

High-grade (Clavien-Dindo

grade $\geq \mathrm{III})$

Bleeding related

Urine leakage

Mortality

6-month preservation rate of

eGFR ${ }^{\ddagger}$

Median, \% (IQR)
$92(85-100)$
$39(48)$

$51(63)$

0.50

41

(55)

0.28

31

(41)

$0.12 \quad 2(3)$

0.027

$6(5-7)$

$12(15)$

$<0.001 \quad 1(3)$

$5(5-$

6)

\section{$\begin{array}{ll}\text { CRO- } & \text { p- } \\ \text { LPN } & \text { value }\end{array}$ \\ group}

75

212

(184-

237)

$20<0.001$

(16-

28)

119
$(40-$

0.002

300) 3-month postoperative

complication, n (\%)

$4(2)$

7 (9)

$0.013 \quad 1(1)$

50

(67)

0.091

38

(51)

0.16

(3) $6(8) \quad 0.14$




\begin{tabular}{|c|c|c|c|c|c|c|}
\hline$\geq 90 \%, \mathrm{n}(\%)$ & $129(61)$ & $37(46)$ & 0.011 & $\begin{array}{l}50 \\
(67)\end{array}$ & $\begin{array}{l}36 \\
(48)\end{array}$ & 0.016 \\
\hline$\geq 80 \%, \mathrm{n}(\%)$ & $185(88)$ & $68(84)$ & 0.23 & $\begin{array}{l}66 \\
(88)\end{array}$ & $\begin{array}{l}63 \\
(84)\end{array}$ & 0.16 \\
\hline$\geq 70 \%, \mathrm{n}(\%)$ & 206 (98) & $77(95)$ & 0.15 & $\begin{array}{l}74 \\
(99)\end{array}$ & $\begin{array}{l}71 \\
(95)\end{array}$ & 0.18 \\
\hline $\begin{array}{l}\text { Median 6-month decrease of } \\
\text { eGFR, } \mathrm{mL} / \mathrm{min} / 1.73 \mathrm{~m}^{2}(\mathrm{IQR})\end{array}$ & $5(0-10)$ & $7(4-12)$ & 0.001 & $\begin{array}{l}5(0- \\
8)\end{array}$ & $\begin{array}{l}7(4- \\
12)\end{array}$ & 0.002 \\
\hline $\begin{array}{l}\text { Upstaging of chronic kidney } \\
\text { disease, } n(\%)\end{array}$ & $50(24)$ & $22(27)$ & 0.33 & $\begin{array}{l}17 \\
(23)\end{array}$ & $\begin{array}{l}20 \\
(27)\end{array}$ & 0.35 \\
\hline $\begin{array}{l}\text { Trifecta achievement rate, } \mathrm{n} \\
(\%)\end{array}$ & 165 (79) & $54(67)$ & 0.027 & $\begin{array}{l}63 \\
(84)\end{array}$ & $\begin{array}{l}48 \\
(64)\end{array}$ & 0.004 \\
\hline
\end{tabular}

${ }^{\dagger}$ interquartile range; ${ }^{\ddagger}$ estimated glomerular filtration rate; ${ }^{\S}$ cortical renorrhaphy omitting- robot-assisted partial nephrectomy; ${ }^{\circledR}$ cortical renorrhaphy omitting- laparoscopic partial nephrectomy.

\section{Figures}


(A)

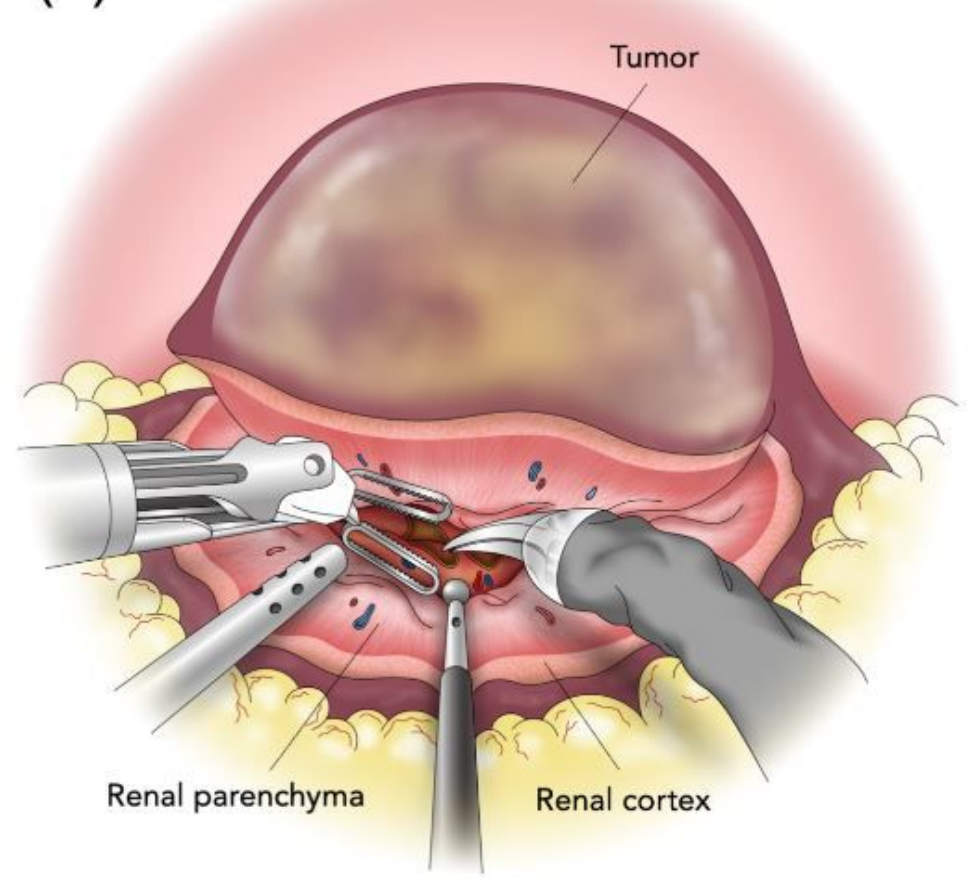

(C)

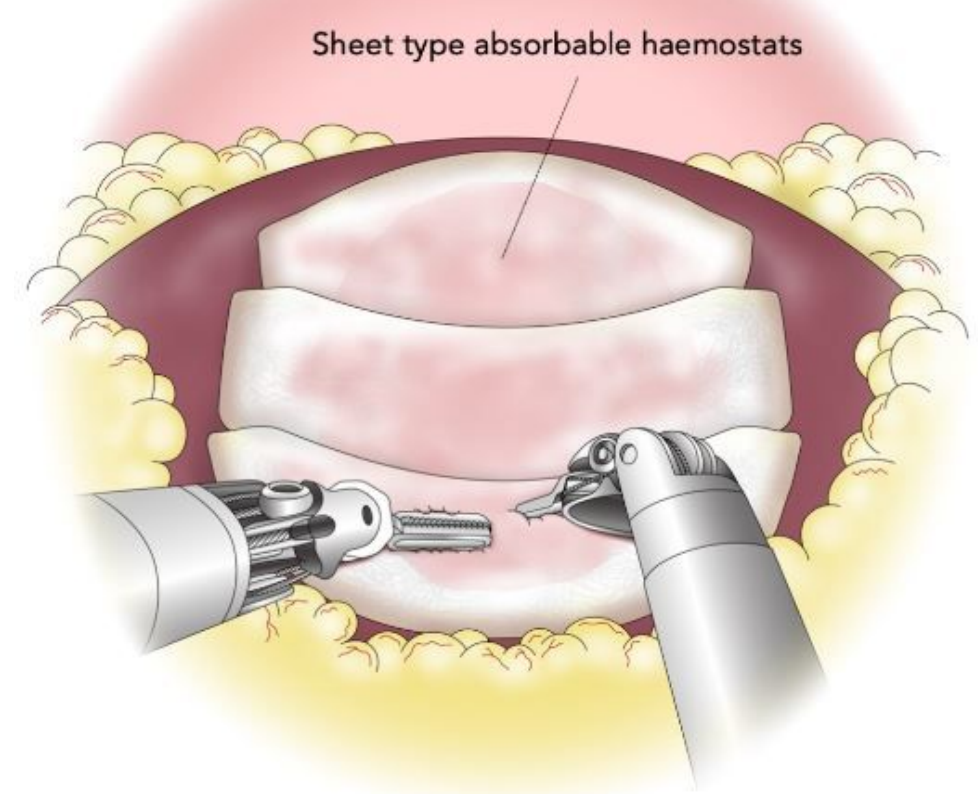

(B)

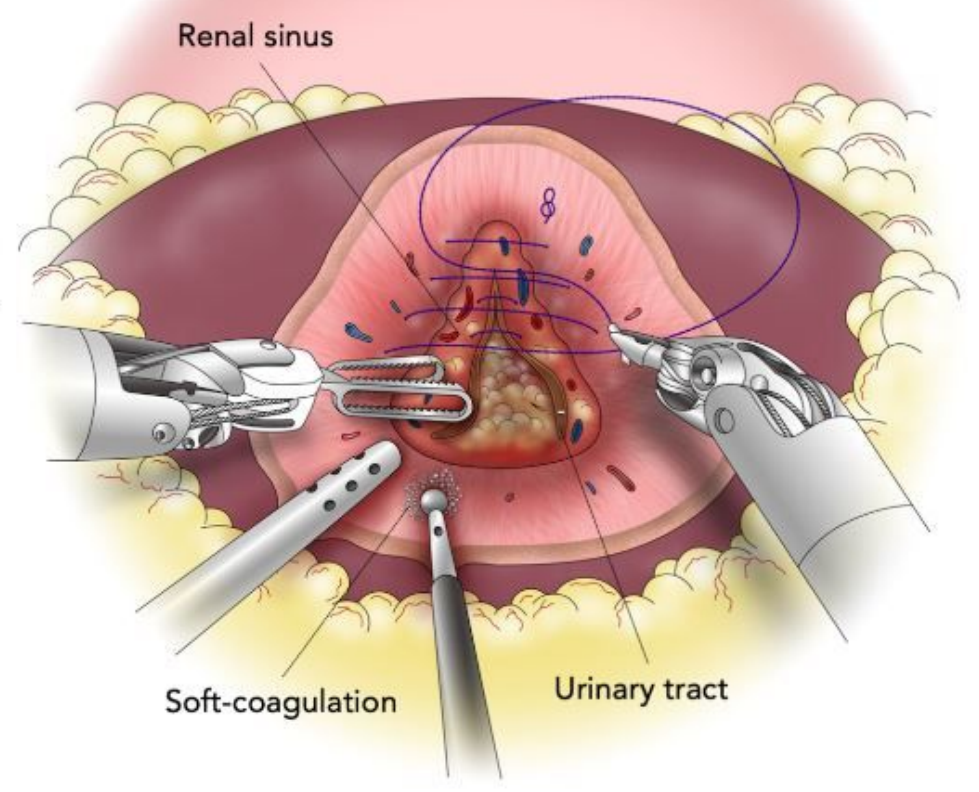

\section{Figure 1}

Surgical procedure omitting cortical renorrhaphy during partial nephrectomy in this study. If the renal sinus or urinary tract is opened, an inner suture is performed (B). In cases without intervention of the renal sinus or urinary tract, the inner suture is omitted and the operation is completed without suturing (only (A) and (C)). (A) Tumor resection technique; renal parenchyma is bluntly dissected along with fiber lines, and bleeding from the cortical or parenchymal vasculature were controlled by soft coagulation. (B) Inner suture technique; opened renal sinuses or urinary collecting systems are closed by running a suture 
parenchymal-layer mediated shallow stitch by 3-0 V-LOC 180 V20 (Covidien, New Haven, CT, USA). Renal cortical hemostasis; cortical renorrhaphy is omitted, and sheet type absorbable hemostats (TachoSil; CSL Behring) are onlayed on the tumor bed.

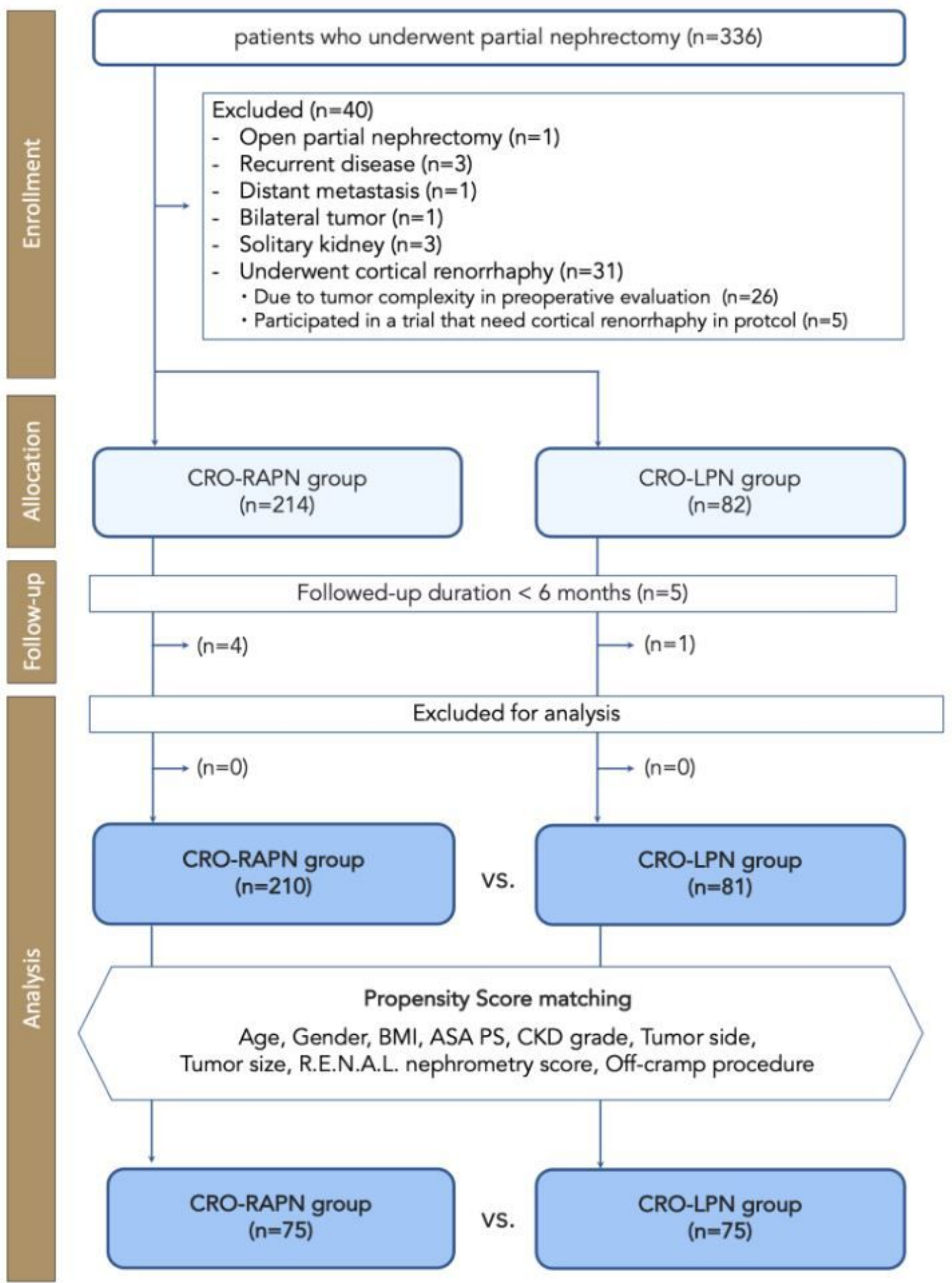

Figure 2

Flow diagram of patient enrolment. 